\title{
Effect of Low-Frequency rTMS and NMES on Subacute Unilateral Hemispheric Stroke With Dysphagia
}

\author{
Kill-Byung Lim, MD, Hong-Jae Lee, MD, Jeehyun Yoo, MD, Yong-Geol Kwon, MD \\ Department of Physical Medicine and Rehabilitation, Inje University Ilsan Paik Hospital, Goyang, Korea
}

Objective To investigate the effect of low-frequency repetitive transcranial magnetic stimulation (rTMS) and neuromuscular electrical stimulation (NMES) on post-stroke dysphagia.

Methods Subacute ( $<3$ months), unilateral hemispheric stroke patients with dysphagia were randomly assigned to the conventional dysphagia therapy (CDT), rTMS, or NMES groups. In rTMS group, rTMS was performed at $100 \%$ resting motor threshold with $1 \mathrm{~Hz}$ frequency for 20 minutes per session ( 5 days per week for 2 weeks). In NMES group, electrical stimulation was applied to the anterior neck for 30 minutes per session (5 days per week for 2 weeks). All three groups were given conventional dysphagia therapy for 4 weeks. We evaluated the functional dysphagia scale (FDS), pharyngeal transit time (PTT), the penetration-aspiration scale (PAS), and the American Speech-Language Hearing Association National Outcomes Measurement System (ASHA NOMS) swallowing scale at baseline, after 2 weeks, and after 4 weeks.

Results Forty-seven patients completed the study; 15 in the CDT group, 14 in the rTMS group, and 18 in the NMES group. Mean changes in FDS and PAS for liquid during first 2 weeks in the rTMS and NMES groups were significantly higher than those in the CDT group, but no significant differences were found between the rTMS and NMES group. No significant difference in mean changes of FDS and PAS for semi-solid, PTT, and ASHA NOMS was observed among the three groups.

Conclusion These results indicated that both low-frequency rTMS and NMES could induce early recovery from dysphagia; therefore, they both could be useful therapeutic options for dysphagic stroke patients.

Keywords Stroke, Dysphagia, Repetitive transcranial magnetic stimulation, Electrical stimulation, Rehabilitation

Received May 13, 2014; Accepted July 14, 2014

Corresponding author: Yong-Geol Kwon

Department of Physical Medicine and Rehabilitation, Inje University Ilsan Paik Hospital, 170 Joowha-ro, Ilsanseo-gu, Goyang 411-706, Korea Tel: +82-31-910-7440, Fax: +82-31-910-7746, E-mail: 19480@paik.ac.kr

(ㄷ) This is an open-access article distributed under the terms of the Creative Commons Attribution Non-Commercial License (http://creativecommons. org/licenses/by-nc/3.0) which permits unrestricted noncommercial use, distribution, and reproduction in any medium, provided the original work is properly cited.

Copyright $\odot 2014$ by Korean Academy of Rehabilitation Medicine

\section{INTRODUCTION}

The cerebral cortex, the swallowing center, various motor and sensory nerves, and anatomical structure cooperate in the human body to deliver the food taken from the mouth to the stomach. In addition to brainstem damage, bilateral, unilateral cerebral lesion, structural injury or damage related to swallowing function may cause dysphagia. Dysphagia, often accompanied by muscle 
weakness after stoke, occurs in $45 \%-65 \%$ of acute stroke patients $[1,2]$. Most patients recover their swallowing function within a few weeks after stroke. This recovery from dysphagia after stroke might follow the reorganization of the non-injured motor cortex, but the extent of recovery varies widely from patient to patient [3]. Dysphagia may cause serious complications, such as aspiration pneumonia, dehydration, malnutrition, or even death. Thus, following early detection of swallowing dysfunction, immediate and appropriate treatment is needed to prevent possible complications caused by dysphagia.

For the rehabilitation of dysphagia, various treatment methods have been used to improve the swallowing function of patients, including sensory stimulation of the oral and facial areas, oral and pharyngeal muscle strengthening, compensatory techniques, and thermal tactile stimulation $[4,5]$. Repetitive transcranial magnetic stimulation (rTMS), a non-invasive method of stimulating the brain, has been used recently as it is known to be effective in controlling the excitability of the cortex and in reducing the imbalance between the hemispheres after stroke $[6,7]$. Neuromuscular electrical stimulation (NMES) has also been used in many studies for the treatment of dysphagia [8-12]. It uses surface electrodes to deliver electrical stimulation to anterior neck muscles causing muscle contraction by depolarizing the nerve fibers within the region of application.

rTMS and NMES are treatment methods commonly used in clinical practice for dysphagia of stroke patients. Michou et al. [13] compared the effect of the 5 Hz-rTMS, the pharyngeal electrical stimulation, and the combined use of the two modalities in patients with chronic dysphagic stroke to that of the control group. However, they used the intraluminal catheter inserted either transnasally or transorally to stimulate the pharyngeal muscles, which was difficult to apply in actual clinical practice. In addition, only single stimulation session was used in that study. Furthermore, the evaluation was conducted immediately and 30 minutes after the session to observe the short-term change. rTMS and NMES are often used clinically during the subacute phase rather than the chronic phase of stroke. NMES with surface electrodes has been widely used in clinical setting because surface electrodes are non-invasive which can be easily applied. Therefore, this study was designed to compare the treatment effects of rTMS and NMES and to follow-up patients with sub- acute unilateral cerebral infarction or hemorrhage with dysphagia.

\section{MATERIALS AND METHODS}

\section{Subjects}

Stroke patients with dysphagia were enrolled in this study. Participant selection criteria were 1) primary diagnosis of unilateral cerebral infarction or hemorrhage with computerized tomography or magnetic resonance imaging scan; 2) stroke onset $<3$ months; 3 ) patients who could maintain their balance during the evaluation and treatment session; and 4) patients who had cognitive function enough to cooperate in the treatment (>15-point on the Korean version of Mini-Mental State Examination [K-MMSE]). Dysphagia was confirmed using a videofluoroscopic swallowing study (VFSS) for the subjects who had clinical symptoms, such as difficulty of swallowing as well as chocking, coughing, wet voice or nasal regurgitation after swallowing. Exclusion criteria for the trial were 1) patients who could not undergo the VFSS or who failed the examination; 2) presence of dysphagia before stroke; 3) history of prior stroke, epilepsy, tumor, radiotherapy in the head and neck, or other neurological diseases; 4) unstable medical condition; and 5) contraindication to magnetic or electrical stimulation.

\section{Study design and evaluation}

Eligible patients with confirmed dysphagia based on the result of the VFSS were randomly allocated to the conventional dysphagia treatment (CDT) group, the rTMS group, or the NMES group using a randomization table. All patients in the three groups received the conventional dysphagia treatment including oropharyngeal muscle-strengthening, exercise for range of motion of the neck and tongue, thermal tactile stimulation, Mendelson maneuver, and food intake training for 4 weeks. The two groups other than the CDT group underwent either rTMS or NMES for two weeks (Fig. 1).

\section{Measurements}

To compare the treatment effects of the three groups, the total score in the functional dysphagia scale (FDS), the pharyngeal transit time (PTT), and the penetrationaspiration scale (PAS) were evaluated using the VFSS at baseline, after 2 weeks, and after 4 weeks. The American 


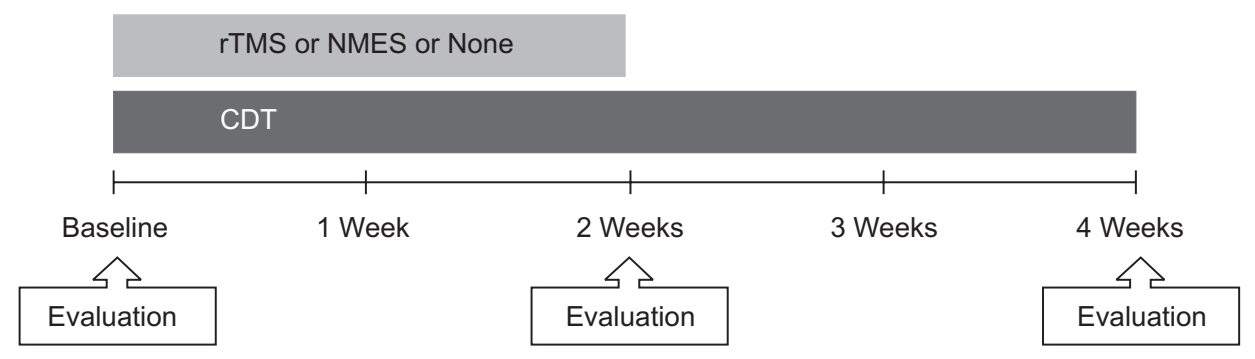

Fig. 1. Intervention design. In rTMS group, rTMS is performed for 20 minutes per session, 5 days per week during the first 2 weeks. In NMES group, electrical stimulation is applied for 30 minutes per session, 5 days per week during the first 2 weeks. All three groups are given CDT for 4 weeks. CDT, conventional dysphagia therapy; rTMS, repetitive transcranial magnetic stimulation; NMES, neuromuscular electrical stimulation.

Speech-Language Hearing Association National Outcomes Measurement System (ASHA NOMS) swallowing scale was assessed. The FDS is based on a physiological scale and is presented as $0-100$ points, depending on the severity of dysphagia [14]. The PTT is one of the sub-items of the FDS, which is a quantitative measurement. The PAS is an 8-point scale that measures selected aspects of penetration and aspiration, conveying depth of airway invasion and whether material entering the airway is expelled [15]. The ASHA NORM swallowing scale is a 7-point rating scale whose lower level indicates severer dysphagia. It was clinically evaluated by integrating all the information from the diet level and the supervision level required [16].

\section{VFSS}

VFSS is a beneficial assessment tool for visualizing the swallow physiology of the oral cavity, pharynx, larynx, and upper esophagus during deglutition. VFSS can directly confirm aspiration and diagnose the anatomical or functional problems of patients. VFSS can be easily conducted, and the appropriate type of diet, education of posture, or need for tube feeding can be determined for individual patients according to their result. Thus, VFSS is commonly utilized in clinical setting to evaluate dysphagia and aspiration [17]. In this study, VFSS was performed in all subjects, with the same protocol used in the fluoroscopic laboratory. Both the lateral and posteroanterior images were obtained following oral administration of $5 \mathrm{~mL}$ semi-solid and $5 \mathrm{~mL}$ liquid bolus (viscosity and material were standardized) while the subjects were seated. One rehabilitation physician with considerable experience performed the VFSS examination and determined the PPT as well as the FDS and PAS scores using videofluoroscopic analysis. He was blinded during this study period by the way of not being informed about the study and grouping of the patients.

\section{rTMS}

Before performing rTMS, we evaluated motor-evoked potentials using Magstim 200 (Magstim, Whiteland, UK) and a coil stimulator. The potentials in the mylohyoid muscle were recorded using an electromyography device (Medelec Synergy, Oxford, UK). The magnetic stimulation was repeated with a change in intensity. The minimum intensity that showed $100 \mu \mathrm{V}$ or more amplitude in three of the five consecutive stimulations was determined as the resting motor threshold [18]. The location yielding the largest response amplitude in the pharyngeal motor cortex of the contralesional hemisphere was determined as a hot spot. For the treatment, $1 \mathrm{~Hz}$ magnetic stimulation at $100 \%$ intensity of the resting motor threshold in the hot spot was applied for 20 minutes each (total 1,200 pulses a day), 5 times a week for 2 weeks.

\section{NMES}

NMES was applied using a modified hand-held batterypowered electrical stimulator VitalStim (Chattanooga Group, Hixson, TN, USA) for 30 minutes per day, 5 times a week, for a total of 2 weeks. The equipment used two independent channel methods with alternating currents. Two sets of electrodes were attached between the digastrics muscle and the hyoid bone and between the hyoid bone and the thyroid cartilage for channel 1 , and between the thyroid cartilage and the cricoids cartilage and vertically under the cricoid cartilage for channel 2. Through this method, we were able to stimulate the muscles needed for swallowing, such as the digastric, 
mylohyoid, and thyrohyoid muscles. The waveform produced by the stimulator was a rectangular symmetric biphasic wave mode. The pulse width and the frequency were determined at $300 \mu \mathrm{s}$ and $80 \mathrm{~Hz}$ with, $100 \mu \mathrm{s}$ in interstimulus intervals. The intensity was between $7 \mathrm{~mA}$ and 9 $\mathrm{mA}$, depending on the compliance of the subjects.

\section{Analysis method}

The changes in the evaluation parameters from the initial to the 2nd week or 4 th week evaluation were analyzed using the Kruskal-Wallis test to determine the statistical difference among three groups. For post-hoc, the Bonferroni method was used. Mann-Whitney U tests were performed in each category between the groups. The significance level of the post-hoc was $0.05 / 3=0.017$. Perprotocol (PP) analysis was performed using the data of the subjects who completed the entire four-week study period, and the intention-to-treat (ITT) analysis was also performed using the data of all the subjects who completed or withdrew from the study, along with the last observation carried forward method. All statistical analyses were performed using the SPSS ver. 18.0 for Windows (SPSS Inc., Chicago, IL, USA). Statistical significance was considered when $\mathrm{p}$-value was less than 0.05 .

\section{RESULTS}

Sixty subjects were randomized into the three aforementioned groups. However, five subjects in the CDT group, six in the rTMS group, and two in the NMES group withdrawn from the study due to follow-up loss at the fourth-week evaluation point. In the PP analysis, the mean age of the CDT group was $62.5 \pm 8.2$ years old, with nine males and six females; in the rTMS group, the mean age was $59.8 \pm 11.8$ years old, with 6 males and 8 females; and in the NMES group, the mean age was $66.3 \pm 15.4$ years old, with 12 males and 6 females. The period from the onset of stroke to the treatment administration was $34.4 \pm 10.1$ days in the CDT group, $30.3 \pm 14.8$ days in the rTMS group, and $37.3 \pm 16.1$ days in the NMES group. In the ITT analysis, the mean age in the CDT group was $60.6 \pm 7.7$ years old, with 13 males and 7 females; in the rTMS group, the mean age was $61.8 \pm 10.4$ years old, with 9 males and 11 females; and in the NMES group, the mean age was $65.4 \pm 15.5$ years old, with 12 males and 8 females. The period from the onset of stroke to the treatment administration was $34.6 \pm 12.1$ days in the CDT group, $32.0 \pm 13.9$ days in the rTMS group, and $37.5 \pm 15.7$ days in the NMES group. The age, sex, type of stroke, location of lesion, score in the K-MMSE and the National Institute of Health Stroke Scale, and period from the onset of stroke to the treatment administration in both the PP and ITT analysis were not significantly different among the three groups at the initial evaluation (Table 1). The FDS, PTT, PAS, and ASHA NOMS scores at the initial evaluation were not significantly different either among the three groups (Table 2).

Table 1. Biomedical characteristics of the subjects

\begin{tabular}{|c|c|c|c|c|c|c|c|c|}
\hline & \multicolumn{4}{|c|}{ Per-protocol analysis } & \multicolumn{4}{|c|}{ Intention-to-treat analysis } \\
\hline & CDT & rTMS & NMES & p-value & CDT & rTMS & NMES & p-value \\
\hline No. of subjects & 15 & 14 & 18 & - & 20 & 20 & 20 & - \\
\hline Age (yr) & $62.5 \pm 8.2$ & $59.8 \pm 11.8$ & $66.3 \pm 15.4$ & 0.127 & $60.6 \pm 7.7$ & $61.8 \pm 10.4$ & $65.4 \pm 15.5$ & 0.125 \\
\hline Gender (male:female) & $9: 6$ & $6: 8$ & $12: 6$ & 0.390 & $13: 7$ & $9: 11$ & $12: 8$ & 0.414 \\
\hline $\begin{array}{l}\text { Type of stroke } \\
\text { (infarction:hemorrhage) }\end{array}$ & 10:5 & $4: 10$ & $8: 10$ & 0.117 & $12: 8$ & $6: 14$ & $10: 10$ & 0.126 \\
\hline Lesion location (left:right) & $7: 8$ & $8: 6$ & $7: 11$ & 0.590 & $9: 11$ & $12: 8$ & $7: 13$ & 0.280 \\
\hline NIHSS & $8.4 \pm 4.3$ & $8.6 \pm 3.4$ & $9.2 \pm 4.0$ & 0.876 & $8.3 \pm 3.8$ & $8.4 \pm 3.6$ & $8.9 \pm 4.1$ & 0.540 \\
\hline K-MMSE & $21.1 \pm 4.0$ & $20.4 \pm 4.8$ & $19.3 \pm 3.7$ & 0.523 & $20.4 \pm 3.9$ & $20.6 \pm 4.7$ & $19.6 \pm 3.8$ & 0.506 \\
\hline Days from stroke & $34.4 \pm 10.1$ & $30.3 \pm 14.8$ & $37.3 \pm 16.1$ & 0.244 & $34.6 \pm 12.1$ & $32.0 \pm 13.9$ & $37.5 \pm 15.7$ & 0.450 \\
\hline
\end{tabular}

Values are presented as number or mean \pm standard deviation.

CDT, conventional dysphagia therapy; rTMS, repetitive transcranial magnetic stimulation; NMES, neuromuscular electrical stimulation; NIHSS, National Institutes of Health Stroke Scale; K-MMSE, Korean Mini-Mental State Examination. 
Table 2. Initial evaluation of the swallowing function

\begin{tabular}{|c|c|c|c|c|c|c|c|c|}
\hline & \multicolumn{4}{|c|}{ Per-protocol analysis } & \multicolumn{4}{|c|}{ Intention-to-treat analysis } \\
\hline & CDT & rTMS & NMES & p-value & CDT & rTMS & NMES & p-value \\
\hline \multicolumn{9}{|l|}{ FDS } \\
\hline Semi-solid & $34.3 \pm 8.7$ & $29.8 \pm 9.8$ & $32.1 \pm 13.2$ & 0.536 & $34.5 \pm 9.0$ & $31.5 \pm 10.7$ & $32.2 \pm 12.8$ & 0.592 \\
\hline Liquid & $52.8 \pm 10.9$ & $47.8 \pm 12.7$ & $51.7 \pm 18.0$ & 0.655 & $51.2 \pm 10.8$ & $48.9 \pm 14.9$ & $51.3 \pm 17.6$ & 0.931 \\
\hline \multicolumn{9}{|l|}{ PTT } \\
\hline Semi-solid & $1.09 \pm 0.36$ & $1.02 \pm 0.20$ & $1.06 \pm 0.31$ & 0.843 & $1.08 \pm 0.30$ & $1.04 \pm 0.18$ & $1.06 \pm 0.30$ & 0.985 \\
\hline Liquid & $1.10 \pm 0.17$ & $1.07 \pm 0.21$ & $1.09 \pm 0.16$ & 0.708 & $1.12 \pm 0.16$ & $1.08 \pm 0.18$ & $1.09 \pm 0.16$ & 0.711 \\
\hline \multicolumn{9}{|l|}{ PAS } \\
\hline Semi-solid & $3.2 \pm 1.6$ & $2.8 \pm 1.4$ & $3.0 \pm 1.6$ & 0.771 & $3.1 \pm 1.4$ & $3.0 \pm 1.2$ & $2.9 \pm 1.5$ & 0.784 \\
\hline Liquid & $5.6 \pm 1.4$ & $5.2 \pm 1.3$ & $5.5 \pm 1.7$ & 0.650 & $5.3 \pm 1.6$ & $5.1 \pm 1.2$ & $5.5 \pm 1.7$ & 0.806 \\
\hline ASHA NOMS & $3.4 \pm 1.0$ & $3.5 \pm 0.8$ & $3.7 \pm 1.2$ & 0.825 & $3.5 \pm 1.1$ & $3.4 \pm 1.0$ & $3.7 \pm 1.2$ & 0.757 \\
\hline
\end{tabular}

Values are presented as mean \pm standard deviation.

CDT, conventional dysphagia therapy; rTMS, repetitive transcranial magnetic stimulation; NMES, neuromuscular electrical stimulation; FDS, functional dysphagia scale; PTT, pharyngeal transit time; PAS, penetration-aspiration scale; ASHA NOMS, American Speech-Language Hearing Association National Outcomes Measurement System.

\section{FDS}

\section{PP analysis}

The score change $(\Delta)$ in FDS for semi-solid materials was not significantly different between groups from the baseline to the 2 nd week ( $\mathrm{p}=0.792$ ) or to the 4 th week evaluation ( $p=0.962)$. The mean change for liquid from the baseline to the 2 nd and the 4 th week evaluations were $-6.50 \pm 4.41$ and $-14.25 \pm 8.45$ in the CDT group, $-15.15 \pm 9.88$ and $-19.15 \pm 12.95$ in the rTMS group, and $-17.89 \pm 9.61$ and $-21.47 \pm 12.44$ in the NMES group, (Fig. 2A). The changes from the baseline to the 2 nd week showed a statistically significant difference between groups $(p=0.002)$. The rTMS and NMES groups showed significant decreases ( $\mathrm{p}=0.016$ and $\mathrm{p}<0.001$, respectively) compared to the CDT group. However, there was no significant difference between the rTMS and the NMES groups $(\mathrm{p}=0.617)$. The change from the initial evaluation to the 4 th week evaluation did not show a statistically significant difference between the groups ( $\mathrm{p}=0.233$ ).

\section{ITT analysis}

The change in FDS for the semi-solid material did not show a significant difference among the groups from baseline to the 2 nd ( $\mathrm{p}=0.895$ ) or the 4 th week evaluation $(\mathrm{p}=0.828)$. The mean changes in FDS for liquid from the baseline to the 2 nd and the 4 th week evaluation were $-7.95 \pm 4.68$ and $-12.60 \pm 7.35$ in the CDT group, $-14.40 \pm 5.61$ and $-17.20 \pm 11.19$ in the rTMS group, and $-17.20 \pm 9.84$ and
$-20.60 \pm 12.71$ in the NMES group (Fig. 2B). The change from the baseline to the 2nd week evaluation showed a significant difference among groups $(\mathrm{p}=0.002)$. The rTMS and NMES groups showed significant decreases $(\mathrm{p}=0.013$ and $\mathrm{p}=0.001$, respectively) compared to the CDT group. However, the difference between the rTMS and NMES groups was not statistically significant $(\mathrm{p}=0.473)$. There was no significant difference in the change from the initial to the 4 th week evaluation among groups $(\mathrm{p}=0.075)$.

\section{PTT}

The PTT result for semi-solid and liquid decreased in all three groups. However, the changes were not statistically significant in either PP or ITT analyses (Table 3).

\section{PAS}

\section{PP analysis}

The score change in PAS for the semi-solid did not show a significant difference among groups from the baseline to the 2 nd or the 4 th week evaluation ( $\mathrm{p}=0.677$ and $\mathrm{p}=0.978$, respectively). The mean changes in the PAS score for liquid from baseline to the 2nd and the 4th week evaluations were $-0.92 \pm 0.76$ and $2.00 \pm 1.00$ in the CDT group, $-2.08 \pm 1.07$ and $-2.54 \pm 1.34$ in the rTMS group, and $-2.16 \pm 1.42$ and $-2.63 \pm 1.46$ in the NMES group (Fig. 3A). The change from baseline to the 2nd week evaluation showed a significant difference among groups $(\mathrm{p}=0.019)$. The rTMS and NMES groups showed significant de- 

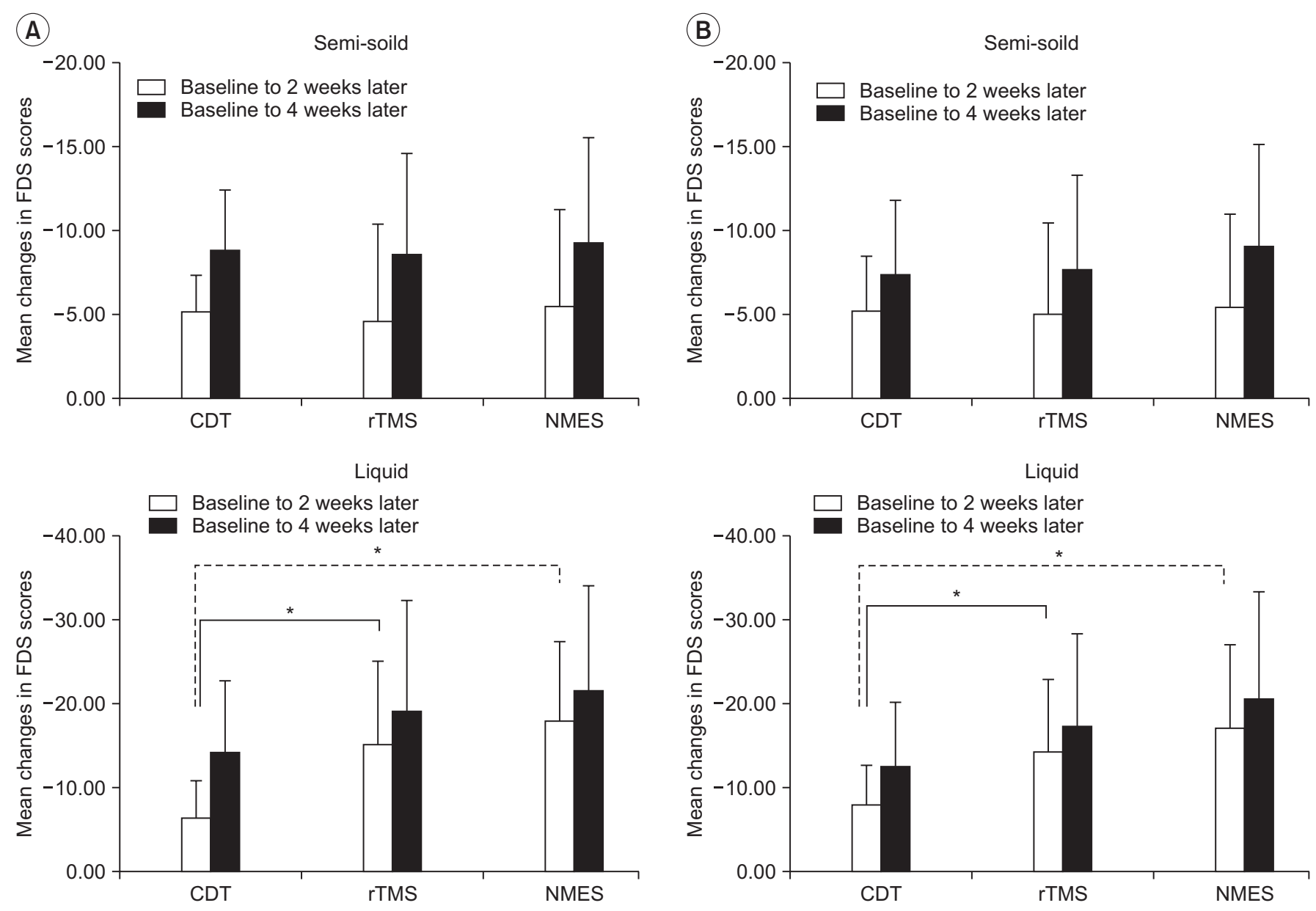

Fig. 2. Mean changes in functional dysphagia scale (FDS) scores by both per-protocol analysis (A) and intention-totreat analysis (B). CDT, conventional dysphagia therapy; rTMS, repetitive transcranial magnetic stimulation; NMES, neuromuscular electrical stimulation. ${ }^{*} \mathrm{p}<0.017$ by the Mann-Whitney U test (vs. CDT group).

Table 3. Comparison of mean changes in pharyngeal transit time

\begin{tabular}{|c|c|c|c|c|c|c|c|c|}
\hline & \multicolumn{4}{|c|}{ Per-protocol analysis } & \multicolumn{4}{|c|}{ Intention-to-treat analysis } \\
\hline & CDT & rTMS & NMES & p-value & CDT & rTMS & NMES & p-value \\
\hline \multicolumn{9}{|l|}{ Semi-solid } \\
\hline$\Delta 0$-2nd week & $-0.04 \pm 0.14$ & $-0.07 \pm 0.12$ & $-0.04 \pm 0.13$ & 0.910 & $-0.05 \pm 0.12$ & $-0.06 \pm 0.11$ & $-0.04 \pm 0.13$ & 0.968 \\
\hline$\Delta 0-4$ th week & $-0.07 \pm 0.24$ & $-0.10 \pm 0.20$ & $-0.08 \pm 0.16$ & 0.819 & $-0.07 \pm 0.19$ & $-0.08 \pm 0.17$ & $-0.08 \pm 0.16$ & 0.791 \\
\hline \multicolumn{9}{|l|}{ Liquid } \\
\hline$\Delta 0-2$ nd week & $-0.02 \pm 0.10$ & $-0.04 \pm 0.09$ & $-0.04 \pm 0.13$ & 0.820 & $-0.04 \pm 0.09$ & $-0.05 \pm 0.09$ & $-0.04 \pm 0.13$ & 0.599 \\
\hline$\Delta 0-4$ th week & $-0.05 \pm 0.13$ & $-0.09 \pm 0.17$ & $-0.06 \pm 0.17$ & 0.833 & $-0.05 \pm 0.11$ & $-0.09 \pm 0.15$ & $-0.06 \pm 0.16$ & 0.638 \\
\hline
\end{tabular}

Values are presented as mean \pm standard deviation.

CDT, conventional dysphagia therapy; rTMS, repetitive transcranial magnetic stimulation; NMES, neuromuscular electrical stimulation.

creases $(\mathrm{p}=0.011$ and $\mathrm{p}=0.014$, respectively) compared to the CDT group. However, the difference between the rTMS and NMES groups was not statistically significant $(p=0.968)$. There was no significant difference in the change from baseline to the 4th week evaluation among groups $(\mathrm{p}=0.540)$. 

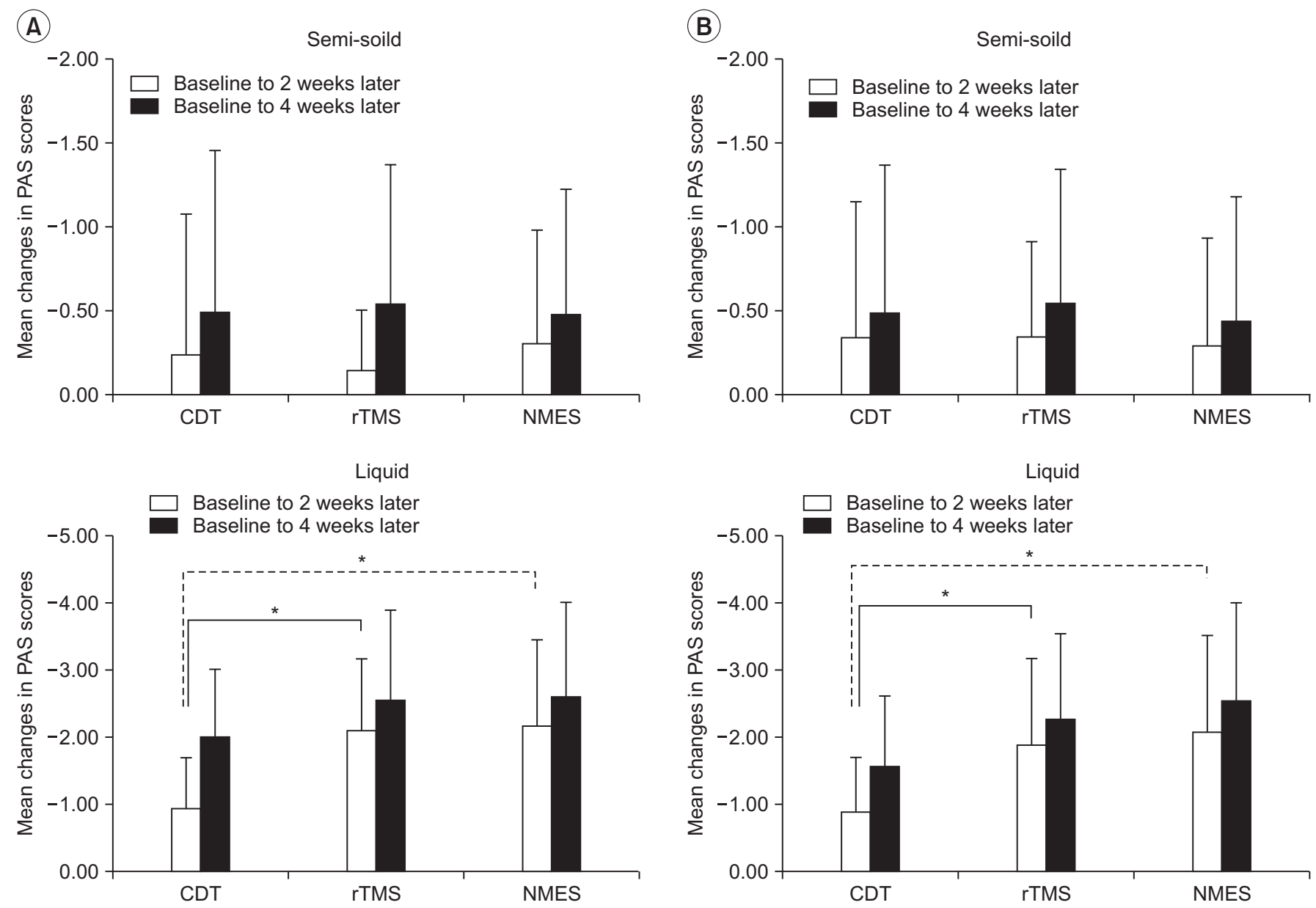

Fig. 3. Mean changes in penetration-aspiration scale (PAS) scores by both per-protocol analysis (A) and intention-totreat analysis (B). CDT, conventional dysphagia therapy; rTMS, repetitive transcranial magnetic stimulation; NMES, neuromuscular electrical stimulation. ${ }^{*} \mathrm{p}<0.017$ by the Mann-Whitney U test (vs. CDT group).

\section{ITT analysis}

The change in the PAS score for the semi-solid did not show a significant difference among groups from the baseline to the 2 nd or the 4 th week evaluations $(\mathrm{p}=0.940$ and $\mathrm{p}=0.860$, respectively). The mean changes in the PAS score for liquid from baseline to the 2nd and the 4th week evaluations were $-0.90 \pm 0.77$ and $1.55 \pm 1.07$ in the CDT group, $1.90 \pm 1.30$ and $-2.25 \pm 1.30$ in the rTMS group, and $-2.10 \pm 1.41$ and $-2.55 \pm 1.47$ in the NMES group (Fig. 3B). The change from the initial to the 2 nd week evaluation showed a significant difference among groups $(\mathrm{p}=0.008$ ). The rTMS and the NMES groups showed significant decreases ( $\mathrm{p}=0.009$ and $\mathrm{p}=0.005$, respectively) compared to the CDT group. However, the difference between the rTMS and the NMES groups was not statistically significant ( $p=0.473)$. There was no significant difference in the change from the initial to the 4th week evaluation among groups $(\mathrm{p}=0.090)$.

\section{ASHA NOMS swallowing scale}

The changes of ASHA NOMs swallowing scale from baseline to the 2 nd and 4 th week evaluations ( $p=0.919$ and $p=0.544$, respectively) did not show significant differences among groups (Fig. 4).

\section{Patient safety}

Potential adverse events from magnetic stimulation, including headache, pain in the administration site, convulsion, decreased hearing ability, nausea, and visual and neurologic changes, were monitored for two weeks. One subject complained of mild headache which was eventually alleviated without any treatment. For the 

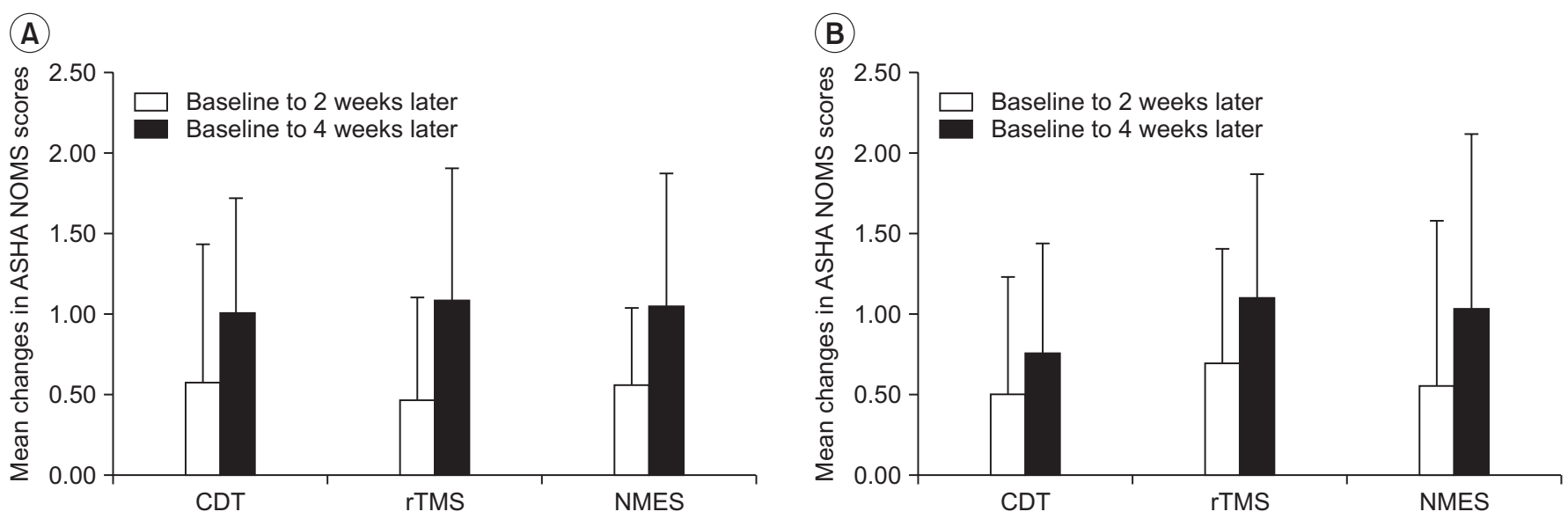

Fig. 4. Mean changes in American Speech-Language Hearing Association National Outcomes Measurement System (ASHA NOMS) swallowing scale by both per-protocol analysis (A) and intention-to-treat analysis (B). CDT, conventional dysphagia therapy; rTMS, repetitive transcranial magnetic stimulation; NMES, neuromuscular electrical stimulation.

NMES group, we checked possible adverse effects, such as the pain or skin irritation in the administration site, laryngospasm, or changes of heart rate and blood pressure. Two subjects complained of mild pain at the administration site, but those symptoms were resolved after the adjustment of the stimulation intensity. There was no subject who complained of other adverse events.

\section{DISCUSSION}

This study was conducted to compare the effects of rTMS and NMES, which are widely used in clinical practice for subacute patients with dysphagia after stroke in the unilateral cerebral hemisphere. Our results showed that the changes in the FDS and PAS scores of the rTMS and NMES groups had statistically significant differences compared to the CDT group for 2 weeks after baseline evaluation, but the differences between the rTMS and the NMES groups were not significant. Other parameters did not show statistical significance among groups.

rTMS is a non-invasive stimulation method to facilitate neurological recovery after stroke. Patients with unilateral brain damage may have an imbalance between the hemispheres. High-frequency magnetic stimulation of an affected hemisphere increases the excitability of the cortex, whereas low-frequency stimulation of the unaffected hemisphere lowers cortical excitability, which might decrease the imbalance between the hemispheres $[6,7]$. However, the exact recovery mechanism of rTMS is currently unclear. Effects of rTMS in stroke patients with dysphagia have been reported in some studies. Verin and Leroi [19] found that, after 5 days of low-frequency (1 $\mathrm{Hz}$ ) rTMS in the unaffected hemisphere, the swallowing function improved significantly in patients who suffered from stroke. Khedr et al. [20] also reported that, after the application of $3 \mathrm{~Hz}-\mathrm{rTMS}$ to the affected hemisphere, a significant improvement in the swallowing function of patients with brain injury compared to the sham-stimulated group. These studies demonstrated the effect of rTMS on dysphagia. However, each study used a different magnetic stimulation frequency with no definitely established protocol. Therefore, the results of those previous studies could not be simply compared. However, Kim et al. [18] conducted a study to compare the $5-\mathrm{Hz}$ stimulation in the affected hemisphere, the $1-\mathrm{Hz}$ stimulation in the unaffected hemisphere, and sham stimulation in patients with brain injury and reported that the FDS and PAS scores showed significant improvement in the low-frequency group compared to the high-frequency or the control groups, with the low-frequency rTMS being effective in improving the swallowing function. It is known that swallowing musculature is discretely and somatotopically represented on the motor and premotor cortex of both hemispheres. It is considered that the dominant hemisphere exerts the principal effect on swallowing function and this dominant hemisphere is independent of handedness [21]. As such, it was reported that patients with a unilateral hemispheric stroke with 
lesions in the dominant hemisphere in terms of the swallowing function can suffer from severe dysphagia, and that the recovery from dysphagia depends on regulating the non-affected hemisphere to reduce interhemispheric inhibition [21]. Based on these factors, our study focused on the function of the unaffected hemisphere to decrease the interhemispheric inhibition and used low-frequency (1 Hz) magnetic stimulation for the rTMS group.

Patients with unilateral hemispheric infarction or hemorrhage were enrolled in this study, but the incidence of dysphasia was $40 \%$ to $80 \%$ in brainstem stroke [22,23]. A previous study has reported that swallowing function is improved in patients with stroke in the brainstem after the application of $3 \mathrm{~Hz}$ magnetic stimulation in the both hemisphere compared to the control group [24]. The mechanism of dysphagia in brainstem stoke includes damage of the corticobulbar tract and disorder of central control due to lesion of the brainstem swallowing center, which is a different from that of hemispheric stroke causing dysphasia. In addition, magnetic stimulation cannot be directly applied in the brainstem. As such, this study excluded the subjects with lesions in the brainstem from the initial study design stage as it used rTMS to decrease the excitability of the contralesional hemisphere so as to decrease the imbalance between the hemispheres.

Many previous studies have reported significant effects of NMES on dysphasia [8-12,25] and suggested that the electrical stimulation could strengthen swallowingrelated muscle and reactivate nerve pathways in the oral cavity. In addition, NMES could increase the local blood flow, reduce the edema, enhance the laryngeal elevation, and protect the striated muscles from the disuse atrophy, which would lead to improved swallowing function $[8,10,26]$. Two mechanisms have been suggested to explain the strengthened swallowing-related muscle seen with NMES. First, the program of a low number of repetitions with a high-intensity muscle contraction increases the muscle mass, which induces augmentation of muscle strength. The second mechanism proposes that the muscle strengthening seen following NMES results from a reversal of voluntary recruitment order with a selective augmentation of type II muscle fibers. Because type II fibers have a higher specific force than type I fibers, selective augmentation of type II muscle fibers will increase the overall strength of the muscle [27]. Our study result that the NMES group showed greater improvement than the CDT group after electrical stimulation for 2 weeks might be due to the selective recruitment of type II fibers than to the increased amount of muscle mass. Another mechanism of NMES suggests that the remodulation of the corticobulbar tract by somatosensory stimulation of the pharynx increases the excitability of swallowing motor cortex $[28,29]$. Considering the short study period used in this study, the positive effect of NMES obtained in this study might be caused by the strengthened muscles rather than by the reactivation of the nerve pathways.

All evaluation parameters showed improvement in all three groups. According to the previous studies on dysphagia after stroke, a large proportion of initially dysphagic patients recover their ability to swallow in the acute stage after the stroke, with prevalence of dysphagia of $47 \%$ at 2 to 3 weeks after the stroke onset, and of $17 \%$ at 2 to 4 months follow-up [30]. About $87 \%$ of the survivors have returned to their pre-stroke diet at 6 months after stroke [31]. As subacute stroke patients were enrolled in this study, the natural recovery from stroke must have also contributed to the observed improvement of the swallowing function. For ethical reasons, the control group had to be given at least the conventional rehabilitation treatment for dysphagia, and as such, it is unclear whether the improvement of swallowing function in our study was due to spontaneous recovery or not. Consequently, it cannot be concluded that all three treatments showed effect on swallowing function even if the evaluation parameters at the 4 th week evaluation exhibited improvement over those at the initial evaluation. The rTMS and NMES groups, however, showed significantly decreased FDS and PAS scores for liquid at 2nd week compared to the CDT group, and the change from baseline to the 4th week did not show a significant difference among groups, indicating that rTMS and NMES could induce early recovery of dysphagia for liquid. Also, the change from the initial to the 2 nd week evaluation did not show a significant difference between the rTMS and NMES groups. The rTMS is central stimulation to control the imbalance between the hemispheres whereas the electrical stimulation applies more to the peripheral nerves and muscles. Even if their mechanisms of action are different, they appear to have the same induction effect for the early recovery of dysphagia for liquid. Considering their different mechanisms, they can be used together for synergic effects in recovering the swallowing function. 
Future studies are required to verify the effectiveness of the combination method.

Two different viscosities-semi-solid and liquid-were used for the VFSS. The PAS score for the semi-solid material after treatment decreased in all three groups compared to that at the baseline evaluation, but the change was not significantly different among groups. This result could be due to the fact that most patients showed lower PAS scores using a semi-solid material than using a liquid material at the initial evaluation, because PAS has only 8 points whereas FDS has a total of 100 points, which might have resulted in the less significant improvement. The PTT showed a decreased pattern in all three groups. The rTMS and NMES groups showed a greater decrease than the CDT group in the PTT, but the difference among the groups was not significant. The PTT is a quantitative variable as one of the sub-items of FDS. Therefore, PTT cannot generally represent the whole swallowing function.

This study was designed to enroll subacute stroke patients who were admitted in our hospital, many of them showed decreased cognitive function. They could not maintain their sitting balance due to severe muscle weakness. As such, the number of potentially eligible patients was limited, which could affect the interpretation of the study results. Some factors, such as their neurologic impairment and age, may influence on the severity of dysphasia, but stratification considering the aforementioned factors was not done at the randomization. Moreover, the study design did not include the sham-stimulation group for both rTMS and NMES, and the CDT group was used as a control group, which led to incomplete blinding. The FDS score is the sum of the scores of the subitems. In this study, only the FDS total score and the PTT were quantitatively assessed, whereas other sub-items in the FDS were not statistically analyzed. Lastly, the swallowing function at 2 and 4 weeks after baseline was evaluated, but long-term follow-up was not performed. Considering the aforementioned limitations, additional studies should be conducted in the future to supplement the results of this study.

In conclusion, this study evaluated the treatment effect of the CDT, rTMS, and NMES in subacute stroke patients. The results of this study demonstrated that both rTMS and NMES induced the early recovery of the swallowing function for liquid in stroke patients, but no difference was observed between the two methods. Therefore, both low-frequency rTMS and NMES might be useful therapeutic options to recover swallowing function of dysphagic stroke patients.

\section{CONFLICT OF INTEREST}

No potential conflict of interest relevant to this article was reported.

\section{REFERENCES}

1. Daniels SK, Brailey K, Priestly DH, Herrington LR, Weisberg LA, Foundas AL. Aspiration in patients with acute stroke. Arch Phys Med Rehabil 1998;79:14-9.

2. Roth EJ, Lovell L, Harvey RL, Heinemann AW, Semik $\mathrm{P}$, Diaz S. Incidence of and risk factors for medical complications during stroke rehabilitation. Stroke 2001;32:523-9.

3. Singh S, Hamdy S. Dysphagia in stroke patients. Postgrad Med J 2006;82:383-91.

4. Langmore S, Miller RM. Behavioral treatment for adults with oropharyngeal dysphagia. Arch Phys Meds Rehabil 1994;75:1154-60.

5. Ludlow CL, Bielamowicz S, Daniels Rosenberg M, Ambalavanar R, Rossini K, Gillespie M, et al. Chronic intermittent stimulation of the thyroarytenoid muscle maintains dynamic control of glottal adduction. Muscle Nerve 2000;23:44-57.

6. Hummel F, Celnik P, Giraux P, Floel A, Wu WH, Gerloff $C$, et al. Effects of non-invasive cortical stimulation on skilled motor function in chronic stroke. Brain 2005;128(Pt 3):490-9.

7. Hummel FC, Cohen LG. Non-invasive brain stimulation: a new strategy to improve neurorehabilitation after stroke? Lancet Neurol 2006;5:708-12.

8. Blumenfeld L, Hahn Y, Lepage A, Leonard R, Belafsky PC. Transcutaneous electrical stimulation versus traditional dysphagia therapy: a nonconcurrent cohort study. Otolaryngol Head Neck Surg 2006;135:754-7.

9. Carnaby-Mann GD, Crary MA. Adjunctive neuromuscular electrical stimulation for treatment-refractory dysphagia. Ann Otol Rhinol Laryngol 2008;117:279-87.

10. Freed ML, Freed L, Chatburn RL, Christian M. Electrical stimulation for swallowing disorders caused by stroke. Respir Care 2001;46:466-74.

11. Kiger M, Brown CS, Watkins L. Dysphagia manage- 
ment: an analysis of patient outcomes using VitalStim therapy compared to traditional swallow therapy. Dysphagia 2006;21:243-53.

12. Suiter DM, Leder SB, Ruark JL. Effects of neuromuscular electrical stimulation on submental muscle activity. Dysphagia 2006;21:56-60.

13. Michou E, Mistry S, Jefferson S, Tyrrell P, Hamdy S. Characterizing the mechanisms of central and peripheral forms of neurostimulation in chronic dysphagic stroke patients. Brain Stimul 2014;7:66-73.

14. Han TR, Paik NJ, Park JW. Quantifying swallowing function after stroke: a functional dysphagia scale based on videofluoroscopic studies. Arch Phys Med Rehabil 2001;82:677-82.

15. Rosenbek JC, Robbins JA, Roecker EB, Coyle JL, Wood JL. A penetration-aspiration scale. Dysphagia 1996;11:93-8.

16. Wesling M, Brady S, Jensen M, Nickell M, Statkus D, Escobar N. Dysphagia outcomes in patients with brain tumors undergoing inpatient rehabilitation. Dysphagia 2003;18:203-10.

17. Groher ME. The detection of aspiration and videofluoroscopy. Dysphagia 1994;9:147-8.

18. Kim L, Chun MH, Kim BR, Lee SJ. Effect of repetitive transcranial magnetic stimulation on patients with brain injury and Dysphagia. Ann Rehabil Med 2011;35:765-71.

19. Verin E, Leroi AM. Poststroke dysphagia rehabilitation by repetitive transcranial magnetic stimulation: a noncontrolled pilot study. Dysphagia 2009;24:204-10.

20. Khedr EM, Abo-Elfetoh N, Rothwell JC. Treatment of post-stroke dysphagia with repetitive transcranial magnetic stimulation. Acta Neurol Scand 2009;119:155-61.

21. Hamdy S, Aziz Q, Thompson DG, Rothwell JC. Physiology and pathophysiology of the swallowing area of human motor cortex. Neural Plast 2001;8:91-7.

22. Meng NH, Wang TG, Lien IN. Dysphagia in patients with brainstem stroke: incidence and outcome. Am J Phys Med Rehabil 2000;79:170-5.

23. Chua KS, Kong KH. Functional outcome in brain stem stroke patients after rehabilitation. Arch Phys Med Rehabil 1996;77:194-7.

24. Khedr EM, Abo-Elfetoh N. Therapeutic role of rTMS on recovery of dysphagia in patients with lateral medullary syndrome and brainstem infarction. J Neurol Neurosurg Psychiatry 2010;81:495-9.

25. Lim KB, Lee HJ, Lim SS, Choi YI. Neuromuscular electrical and thermal-tactile stimulation for dysphagia caused by stroke: a randomized controlled trial. J Rehabil Med 2009;41:174-8.

26. Sun SF, Hsu CW, Lin HS, Sun HP, Chang PH, Hsieh $\mathrm{WL}$, et al. Combined neuromuscular electrical stimulation (NMES) with fiberoptic endoscopic evaluation of swallowing (FEES) and traditional swallowing rehabilitation in the treatment of stroke-related dysphagia. Dysphagia 2013;28:557-66.

27. Lake DA. Neuromuscular electrical stimulation: an overview and its application in the treatment of sports injuries. Sports Med 1992;13:320-36.

28. Fraser C, Power M, Hamdy S, Rothwell J, Hobday D, Hollander I, et al. Driving plasticity in human adult motor cortex is associated with improved motor function after brain injury. Neuron 2002;34:831-40.

29. Gow D, Hobson AR, Furlong P, Hamdy S. Characterising the central mechanisms of sensory modulation in human swallowing motor cortex. Clin Neurophysiol 2004;115:2382-90.

30. Finestone HM, Greene-Finestone LS, Wilson ES, Teasell RW. Malnutrition in stroke patients on the rehabilitation service and at follow-up: prevalence and predictors. Arch Phys Med Rehabil 1995;76:310-6.

31. Mann G, Hankey GJ, Cameron D. Swallowing function after stroke: prognosis and prognostic factors at 6 months. Stroke 1999;30:744-8. 